\title{
Accurate structure determination of nanocrystals by continuous precession electron diffraction tomography
}

\author{
Yihan Shen ${ }^{1 \dagger}$, Wenjia Sun ${ }^{1 \dagger}$, Yang Liu ${ }^{3}$, Zhengyang Zhou ${ }^{1,2^{*}}$ and Junliang Sun ${ }^{1 *}$
}

Structures are the cornerstones of structure-function relationship research of materials. After more than a hundred years of development, single crystal X-ray diffraction (SC-XRD) becomes the most common method to determine structures of materials. Nevertheless, the weak interaction between X-ray and matter requires crystals to be larger than $5 \mu \mathrm{m} \times 5 \mu \mathrm{m} \times 5 \mu \mathrm{m}$ for normal SC-XRD diffractometer. Recent appearances of X-ray free electron lasers (XFEL) and micro-focused synchrotron beamlines allow SC-XRD data collection from micron-sized crystals, but access to these facilities is limited. The development of three-dimensional (3D) electron diffraction (ED) has provided a powerful tool for scientists to explore the microcosmos, extending the limit of the SC-XRD method [1]. With 3D ED method, the structures of nanocrystals can be determined, and different phases in polycrystal samples can be identified [2-4]. However, the strong interaction between electrons and matters during the ED process breaks the kinematical relationship between diffraction intensity and structure factors. Hence enough confidence cannot be given by the structure solved by
3D ED with the normal data analysis methods of XRD based on kinematical theory.

To improve confidence of structures determined by 3D ED, Palatinus et al. [5,6] developed the dynamical refinement method against 3D ED data collected through precession electron diffraction tomography (PEDT). With the dynamical refinement of PEDT data, it is confident to locate hydrogen atom positions in structures without rigid restraints and to determine absolute configurations for nanocrystals $[7,8]$. However, the step-wise data acquisition process in PEDT usually introduces unnecessary electron dose which could damage the crystallinity of beam-sensitive samples [9], such as metal organic frameworks (MOFs), covalent organic frameworks (COFs), major organic compounds, and nano-catalysts [9]. The continuous rotation ED (cRED) method, in which the rotation of sample is continuous during the exposure of camera, is developed to solve this problem [9]. However, the diffraction geometries of PEDT and cRED are very different (Fig. 1a, b). The dynamical refinement method developed based on the diffrac-
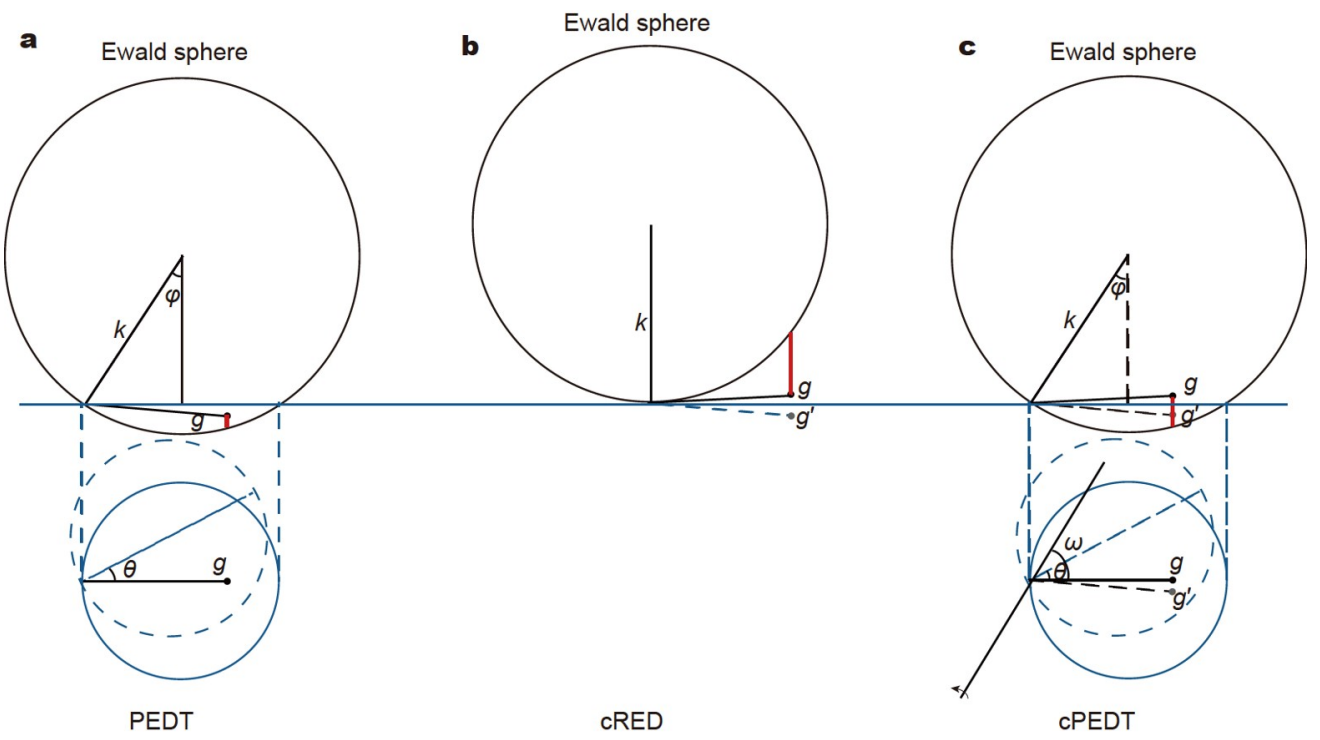

Figure 1 The geometry of PEDT (a), cRED (b), and cPEDT (c). The $S$ (the red line) will change during the rotation of the crystal. The bottom views of (a, c) are normal to the optical axis of the transmission electron microscope (TEM).

\footnotetext{
${ }^{1}$ State Key Laboratory of Rare Earth Materials Chemistry and Applications, College of Chemistry and Molecular Engineering, BNLMS, Peking University, Beijing 100871, China

${ }^{2}$ Shanghai Institute of Ceramics, Chinese Academy of Sciences, Shanghai 201899, China

${ }^{3}$ ReadCrystal Biotech., Suzhou 215500, China

$\dagger$ These authors contributed equally to this work.

* Corresponding authors (emails: zhouzhengyang@mail.sic.ac.cn (Zhou Z); junliang.sun@pku.edu.cn (Sun J))
} 
tion geometry of PEDT cannot be applied to cRED data. Here, we present a new 3D ED data collection method named continuous PEDT (cPEDT), which is the combination of PEDT and cRED. With cPEDT method, unnecessary electron doses between the step-wise tilts during PEDT can be avoided, and the dynamical refinement can be realized.

In CPEDT (Fig. 1c), the precession of the incident beam makes Ewald sphere tilt for an angle $\varphi$ and rotate along the $z$ axis with precession phase $\theta$, while the continuous rotation makes the reciprocal lattice rotate continuously along an axis vertical to $z$ axis with angle $\alpha$. If the rotation angle during the exposure time of one frame is $\alpha_{\max }$, the tilting of the reciprocal lattice will be from $-\alpha_{\max } / 2$ to $\alpha_{\max } / 2$. The excitation error $S$ changes during the precession of the beam and the rotation of the sample, so the intensity of diffraction changes. The intensity of diffraction $\left(I_{\mathrm{tb}}\right)$ with $S$ can be approximated by the two-beam expression [10]:

$I_{\mathrm{tb}}=\left(\frac{\pi t}{\xi}\right)^{2 \sin ^{2}\left[\pi t\left(S^{2}+\xi^{-2}\right)\right]} \frac{\left.\pi t\left(S^{2}+\xi^{-2}\right)\right]^{2}}{[\pi}$

where $t$ is the thickness of the sample, and $\xi$ is the extinction

$$
\left[\begin{array}{cc}
\cos ^{2}(\omega+\theta)(1-\cos \alpha)+\cos \alpha & \cos (\omega+\theta) \sin (\omega+\theta)(1-\cos \alpha) \\
\cos (\omega+\theta) \sin (\omega+\theta)(1-\cos \alpha) & \sin ^{2}(\omega+\theta)(1-\cos \alpha)+\cos \alpha \\
-\sin \alpha \sin (\omega+\theta) & \sin \alpha \cos (\omega+\theta)
\end{array}\right.
$$

The new diffraction vector is $\boldsymbol{g}^{\prime}=\boldsymbol{T} \boldsymbol{g}$. As $\alpha$ is a very small angle (always less than $1^{\circ}$ ), there is $\cos \alpha=1$ and $\sin \alpha=\alpha$. So $\boldsymbol{g}^{\prime}$ has

$$
\begin{aligned}
x_{g^{\prime}} & =g \cos \theta+\alpha \sin (\omega+\theta) z_{g}, \\
y_{g^{\prime}} & =g \sin \theta-\alpha \cos (\omega+\theta) z_{g}, \\
z_{g^{\prime}} & =-\alpha \sin (\omega+\theta) g \cos \theta+\alpha \cos (\omega+\theta) g \sin \theta+z_{g} \\
& =z_{g}-\alpha g \sin \omega .
\end{aligned}
$$

The excitation error $S$ should be the solution of the equation $\left(x_{g^{\prime}}-k_{x}\right)^{2}+y_{g^{\prime}}{ }^{2}+\left(z_{g^{\prime}}+S-k_{z}\right)^{2}=k^{2}$. Negating the square terms of $S$ and $\alpha$, there is

$S=\frac{g^{2}}{2 k}-z_{g}-g \varphi \cos \theta+g \alpha \sin \omega=S_{0}-g \varphi \cos \theta+g \alpha \sin \omega$.

The excitation error of cPEDT has one more term, $g \alpha \sin \omega$, than PEDT. As precession speed is much faster than continuous rotation speed, the intensity of diffraction can be regarded as a double integral over $\theta$ and $\alpha$. Using Equations (1) and (3), the integral intensity of cPEDT becomes

$I_{\mathrm{int}}=\frac{1}{2 \pi \alpha_{\max }} \int_{-\frac{\alpha_{\max }}{2}}^{\frac{\alpha_{\max }}{2}} \int_{0}^{2 \pi} I_{\mathrm{tb}}\left(S_{0}-g \varphi \cos \theta+g \alpha \sin \omega\right) \mathrm{d} \theta \mathrm{d} \alpha$.

To compare the relationship between integral intensity $I_{\text {int }}$ and excitation error $S_{0}$ for PEDT and CPEDT, simulations based on Equation (4) have been done with different $g, \alpha_{\max }=1^{\circ}, \varphi=1^{\circ}, \omega$ $=90^{\circ}$, different $\xi$, different $t$ and a mosaicity of $0.05^{\circ}$ (Figs 2 and $3)$. According to the simulation, the intensities of PEDT and cPEDT have relatively big difference at the local maxima of PEDT, when the absolute value of $S_{0}$ is comparable to or higher than $g \varphi$. However, there are still some ranges of $S_{0}$ where the difference is acceptably small as $\sigma$ of the measured intensity of length of the diffraction.

In PEDT, the excitation error $S$ of a diffraction vector $\boldsymbol{g}=\left[\begin{array}{c}g \cos \theta \\ g \sin \theta \\ z_{g}\end{array}\right]$ can be obtained by solving the equation of $\left(x_{g}-k_{x}\right)^{2}+y_{g}{ }^{2}+\left(z_{g}+S-k_{z}\right)^{2}=k^{2}$. The solution is

$S=\frac{g^{2}}{2 k}-z_{g}-g \varphi \cos \theta=S_{0}-g \varphi \cos \theta$,

where $S_{0}$ is the distance from the diffraction vector to Ewald sphere with no precession. In CPEDT, an $\alpha$-angle tilt axis is applied to the system. The angle between the projection of this axis on the image and the diffraction vector $\boldsymbol{g}$ is $\omega$. As the coordinate system is rotating with the changing of $\theta$, the rotation axis should be described as $\left[\begin{array}{c}\cos (\omega+\theta) \\ \sin (\omega+\theta) \\ 0\end{array}\right]$ and the rotation matrix

$T$ is

$$
\begin{gathered}
\sin \alpha \sin (\omega+\theta) \\
-\sin \alpha \cos (\omega+\theta) \\
\cos \alpha
\end{gathered}
$$

cPEDT andPEDT. If only the diffraction with different $g$ in these ranges of $S_{0}$ is taken into account, the cPEDT data can be processed using the same way as PEDT. As the shape of the $I_{\text {int }}-S_{0}$ plot with the change of $\xi$ or $t$ does not change much (Figs 2 and 3 ), the different ranges of $S_{0}$ for different $g$ should be capable for data collected from samples with various structures and thickness. Moreover, according to Equation (3), data of cPEDT with lower $\alpha_{\max }$ and $\omega \neq \pm 90^{\circ}$ will have less difference with PEDT, which were also proved in the Supplementary information (Figs S1-S4). It means that the data extraction will be more precise when the rotation angle in a frame is smaller, if the data is processed in the same way as PEDT. The appropriate ranges of $S_{0}$ for diffraction with different $g$, which can be applied in cases with $\alpha_{\max }=1^{\circ}$ and $\varphi=1^{\circ}$, are listed in Table S1.

To prove our above simulations, the cPEDT data of $\mathrm{MgMoO}_{4}$ and beam-sensitive organic compound $\mathrm{C}_{16} \mathrm{H}_{17} \mathrm{NO}$ were collected, processed and refined with the dynamical diffraction theory. The PEDT data of the same particle of $\mathrm{MgMoO}_{4}$ and SC$\mathrm{XRD}$ data of $\mathrm{C}_{16} \mathrm{H}_{17} \mathrm{NO}$ were applied as comparisons. The asobtained crystallographic data and refinement details are provided in Tables S2-S5. It can be found that CPEDT can indeed be applied to collect 3D ED data of beam-sensitive materials, and the accurate structures can really be determined with the dynamical refinement of cPEDT data (Figs S5-S7).

In conclusion, a new 3D ED data collection method named cPEDT was developed through the combination of PEDT and cRED. With cPEDT method, unnecessary electron doses between the step-wise tilts during PEDT can be avoided, and accurate structures can be determined with dynamical refinement. Such an advantageous method can promote the determination of fine structural features, hydrogen atom position, absolute configuration, etc., in much more beam-sensitive 

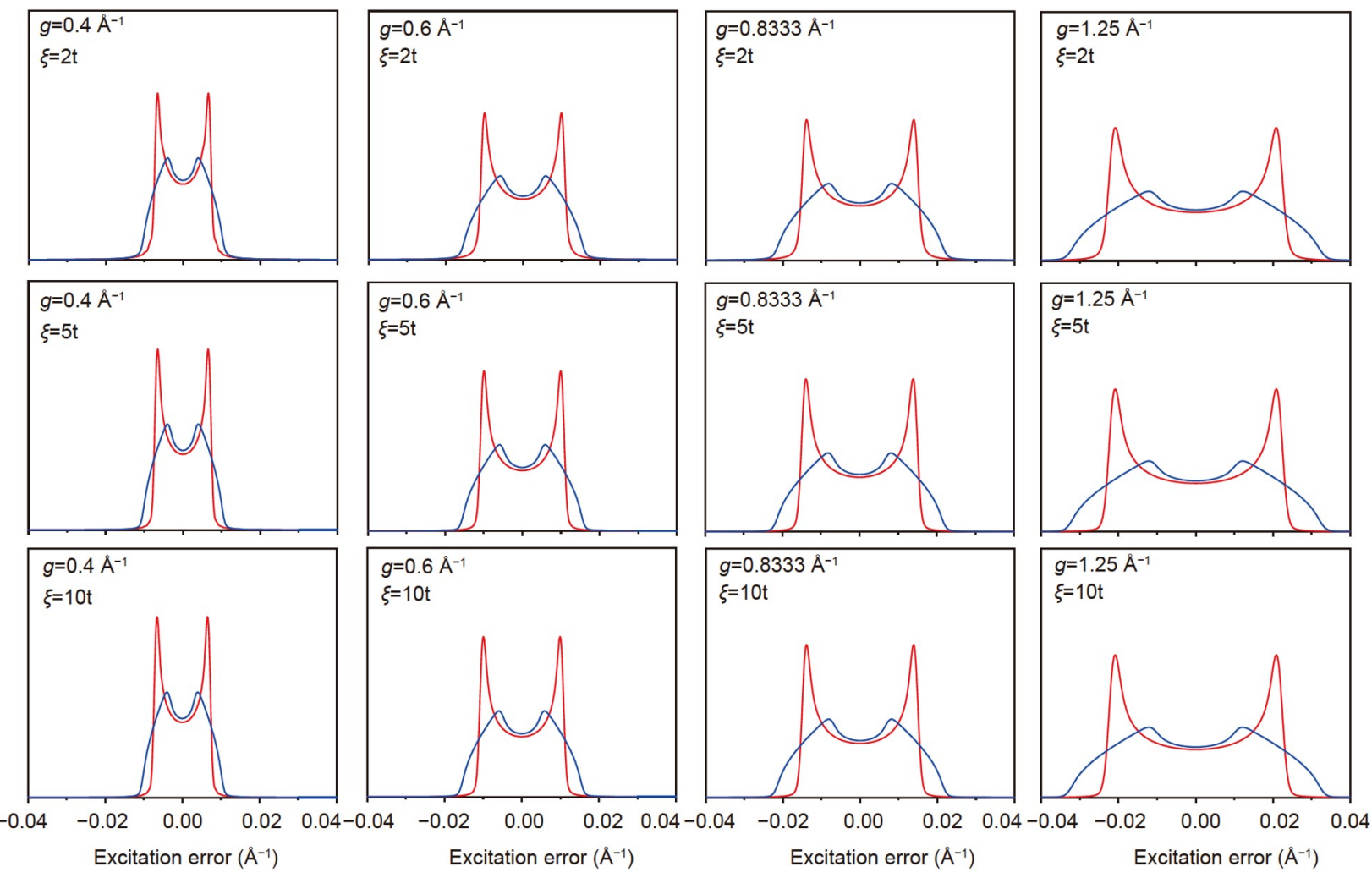

Figure 2 Mosaicity-smeared intensity-excitation error plots with $t=80 \mathrm{~nm}, \varphi=1^{\circ}$ and $\omega=90^{\circ}$. The red plots represent PEDT and the blue plots represent cPEDT.
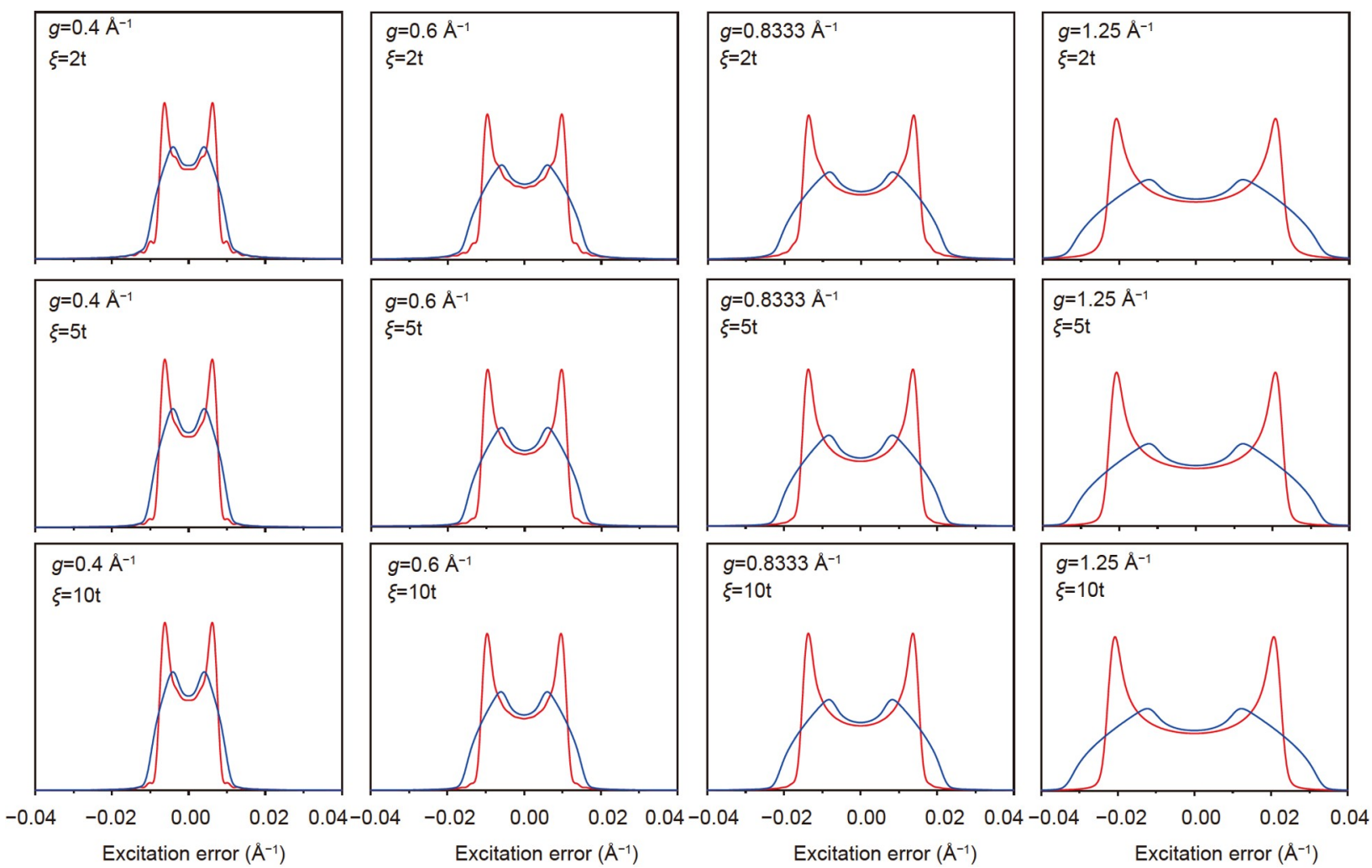

Figure 3 Mosaicity-smeared intensity-excitation error plots with $t=40 \mathrm{~nm}, \varphi=1^{\circ}$ and $\omega=90^{\circ}$. The red plots represent PEDT and the blue plots represent cPEDT. 
materials, such as MOFs, COFs, organic compounds, and nanocatalysts.

Received 2 November 2021; accepted 20 December 2021; published online 17 February 2022

1 Huang Z, Grape ES, Li J, et al. 3D electron diffraction as an important technique for structure elucidation of metal-organic frameworks and covalent organic frameworks. Coord Chem Rev, 2021, 427: 213583

2 Mugnaioli E, Andrusenko I, Schüler T, et al. Ab initio structure determination of vaterite by automated electron diffraction. Angew Chem Int Ed, 2012, 51: 7041-7045

3 Hynek J, Brázda P, Rohlíček J, et al. Phosphinic acid based linkers: Building blocks in metal-organic framework chemistry. Angew Chem Int Ed, 2018, 57: 5016-5019

$4 \mathrm{Li} \mathrm{J}$, Lin C, Min Y, et al. Discovery of complex metal oxide materials by rapid phase identification and structure determination. J Am Chem Soc, 2019, 141: 4990-4996

5 Palatinus L, Petříček V, Corrêa CA. Structure refinement using precession electron diffraction tomography and dynamical diffraction: Theory and implementation. Acta Crystlogr Found Adv, 2015, 71: 235244

6 Palatinus L, Corrêa CA, Steciuk G, et al. Structure refinement using precession electron diffraction tomography and dynamical diffraction: Tests on experimental data. Acta Crystlogr B Struct Sci Cryst Eng Mater, 2015, 71: 740-751

7 Palatinus L, Brázda P, Boullay $\mathrm{P}$, et al. Hydrogen positions in single nanocrystals revealed by electron diffraction. Science, 2017, 355: 166169

8 Brázda P, Palatinus L, Babor M. Electron diffraction determines molecular absolute configuration in a pharmaceutical nanocrystal. Science, 2019, 364: 667-669

9 Gemmi M, Lanza AE. 3D electron diffraction techniques. Acta Crystlogr B Struct Sci Cryst Eng Mater, 2019, 75: 495-504

10 Palatinus L, Brázda P, Jelínek M, et al. Specifics of the data processing of precession electron diffraction tomography data and their implementation in the program PETS2.0. Acta Crystlogr B Struct Sci Cryst Eng Mater, 2019, 75: 512-522

Acknowledgements We cordially thank the Institute of Chemistry, Chinese Academy of Sciences for supply of TEM to realize the PEDT and cPEDT data collection. This work was supported by the National Basic Research Program of China (2020YFA0210700 and 2016YFA0301004), the National Natural Science Foundation of China (21871009, 21527803, 22125102, and 22105218), and Shanghai Sailing Program (21YF1454400).

Author contributions Sun J and Zhou Z conceived and designed the experiments. Shen $\mathrm{Y}$ and Sun W performed all experimental work. Liu Y assisted Shen $\mathrm{Y}$ and Sun $\mathrm{W}$ to collect cPEDT data of the beam-sensitive sample. All the authors discussed the results and commented on the manuscript.

Conflict of interest The authors declare that they have no conflict of interest.

Supplementary information Supporting data are available in the online version of the paper.

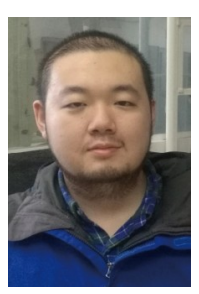

Yihan Shen is currently a $\mathrm{PhD}$ candidate at the College of Chemistry and Molecular Engineering, Peking University. His research interests include the theoretical research of Xray diffraction and electron diffraction.

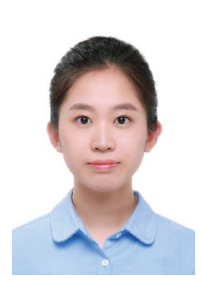

Wenjia Sun is currently a $\mathrm{PhD}$ candidate at the College of Chemistry and Molecular Engineering, Peking University. Her research interests include electron diffraction and its application in nanocrystals.

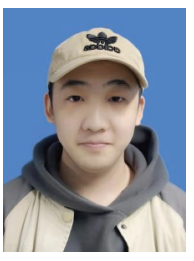

Yang Liu is currently an engineer of ReadCrystal Biotech. His research interest is the application of electron diffraction in nanocrystals.

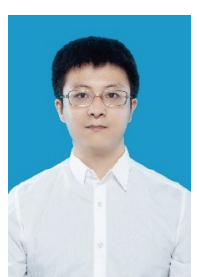

Zhengyang Zhou received his BSc degree (2012) in materials science and engineering from Dalian Polytechnic University and $\mathrm{PhD}$ degree (2018) in chemistry from Chongqing University. Between 2012 and 2018, he was joint-supervised under Prof. Junliang Sun at Peking University. He moved to Shanghai Institute of Ceramics, Chinese Academy of Sciences, and became a full assistant researcher in 2020. His current research interests include the method development for structure determination and exploration of new function ceramics and thermoelectric materials.

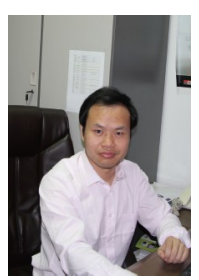

Junliang Sun is a full professor at Peking University. $\mathrm{He}$ received his BSc degree (2001) and $\mathrm{PhD}$ degree (2006) in chemistry from Peking University. After finishing his postdoctoral work at Cornell University and Stockholm University, he became an assistant professor at Stockholm University in 2009. In 2012, he moved to Peking University. His current research interests include the method development for structure determination and synthesis and applications of porous materials and dense oxides.

\section{利用连续旋转旋进电子衍射断层扫描确定纳米晶的 确切结构}

沈弯寒 $^{1 \dagger}$, 孙文甲 ${ }^{1 \dagger}$, 刘扬 $^{3}$, 周钲洋 ${ }^{1,2^{*}}$, 孙俊良 ${ }^{1^{*}}$

摘要 三维电子衍射已逐渐成为人们探索微观世界的强有力的工具. 本文中, 我们展示了一种新的三维电子衍射数据收集方法, 命名为连续 旋转旋进电子衍射断层扫描( $\mathrm{cPEDT}$ ), 该方法是旋进电子衍射断层扫描 (PEDT) 与连续旋转电子衍射 (cRED) 的结合. 使用 $\mathrm{cPEDT}$ 方法, 可以避 免PEDT方法中步进式过程引入的不必要的电子剂量, 同时利用cPEDT 收集到的数据也可以进行动力学精修从而确定材料的精确结构. 该方 法可以促进三维电子衍射在一些电子束敏感材料上的应用, 如金属-有 机框架(MOF)、共价有机框架(COF), 以及一些有机物. 